\title{
MicroRNAs and Esophageal Squamous Cell Carcinoma
}

\author{
Kayoko Matsushima ${ }^{a}$ Hajime Isomoto $^{a}$ Shigeru Kohno ${ }^{b}$ Kazuhiko Nakao $^{a}$

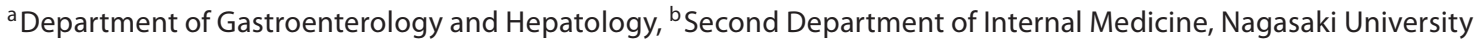 \\ Hospital, Nagasaki, Japan
}

\section{Key Words}

Esophageal cancer $\cdot$ Esophageal squamous cell carcinoma $\cdot$ MicroRNA $\cdot$ miR-205 $\cdot \mathrm{miR}-10 \mathrm{a} \cdot \mathrm{miR}-200$ family $\cdot$

Epithelial-mesenchymal transition

\begin{abstract}
Esophageal cancer is a common cause of cancer death worldwide. Esophageal squamous cell carcinoma (ESCC) is the most predominant type. Certain microRNAs (miRNAs) function in tumorgenesis involved in important biological and pathologic processes. To reveal miRNAs' signatures of ESCC, we analyzed miRNAs extracted from ESCC cell lines with the microRNA microarray. The significant alterations were confirmed by quantitative real-time PCR using miRNAs extracted from cell lines or patients' esophageal biopsy tissues. We found that miR-205 and miR-10a were significantly altered in cellular expression, and might be specific for ESCC with potential roles in the pathogenesis. As a result of function studies in miR-205, alterations in miR-205 expression could modulate the phenotype of epithelial cells towards epithelial-mesenchymal transition characterized by reduced abundance of E-cadherin, that is the ESCC-specific miRNA target and inhibit translationally a representative $\mathrm{E}$-cadherin repressor, ZEB1 and ZEB2. Similarly, miR-10a is reported as a tumor suppressor by controlling cell migration/invasion, as it can target homeobox genes. These results provide insight
\end{abstract}

into the potential mechanisms of ESCC in the pathogenesis. This review also includes a comprehensive overview of the relationship between miRNAs and ESCC.

Copyright ๑ 2010 S. Karger AG, Basel

\section{Introduction}

Esophageal cancer is the eighth most common cancer and the sixth most common cause of cancer deaths worldwide. Although Barrett's adenocarcinoma is the most rapidly increasing cancer in Western countries, esophageal squamous cell carcinoma (ESCC) is still dominant in East Asia [1]. Often diagnosed at later stages of ESCC, the prognoses of affected patients are unsatisfactory despite the development of therapeutic options such as surgery, chemotherapy and radiotherapy. Consequently, there is a great need for biomarkers to allow a tailored multimodality approach with increased efficacy. Nevertheless, to date, efforts to indentify molecular markers in association with the pathogenesis of ESCC have proven to be primarily unsuccessful [2].

MicroRNAs (miRNA) are involved in biological and pathologic processes including cell differentiation, proliferation, apoptosis and metabolism, and are emerging as highly tissue-specific biomarkers with potential clinical applicability for defining cancer types and origins [3].

\section{KARGER \\ Fax +4161306 1234 E-Mail karger@karger.ch} www.karger.com (c) 2010 S. Karger AG, Basel $0012-2823 / 10 / 0823-0138 \$ 26.00 / 0$

Accessible online at: www.karger.com/dig
Hajime Isomoto, MD

Department of Gastroenterology and Hepatology

Nagasaki University Hospital

Nagasaki 852-8501 (Japan)

Tel. +8195819 7481, Fax +8195819 7482, E-Mail hajimei2002@yahoo.co.jp 
Table 1. MicroRNA expression patterns in ESCC in the literature

\begin{tabular}{|c|c|c|c|c|}
\hline \multicolumn{4}{|c|}{ Expression patterns in ESCC } & \multirow{2}{*}{$\begin{array}{l}\text { Other cancers } \\
\text { Involvement }\end{array}$} \\
\hline $\begin{array}{l}\text { microRNA } \\
(\mathrm{miR})\end{array}$ & $\begin{array}{l}\text { compared to } \\
\text { non-malignant } \\
\text { esophageal tissues }\end{array}$ & $\begin{array}{l}\text { compared to } \\
\text { other histological } \\
\text { cancer cell types }\end{array}$ & proved targets & \\
\hline $\operatorname{miR}-21$ & $\uparrow[1,5,9]$ & $\uparrow[9]$ & $\mathrm{PDCD}^{1}{ }^{1}, \mathrm{NFIB}^{2}, \mathrm{PTEN}^{3}, \mathrm{TPM}^{4}$ & $\begin{array}{l}\uparrow \text { breast ca. }{ }^{5} \text {, lung ca., prostate ca., ovarian ca., pancreatic } \\
\text { ca., colon ca., gastric ca., AML }{ }^{6} \text {, aggressive CLL }{ }^{7} \text {, } \\
\text { glioblastoma [4], HCC }{ }^{8} \text {, cholangioca. [9], adenoca. of } \\
\left.\text { esophagus, HNSCC }{ }^{9} 16\right], \text { SCC of tongue }\end{array}$ \\
\hline miR-93 & $\uparrow[5]$ & & FUS1, E2F1 ${ }^{10}$, TP53INP1 $^{11}$ & $\begin{array}{l}\uparrow \text { Gastric ca., HCC } \\
\downarrow \text { ATL }^{12}\end{array}$ \\
\hline miR-373 & $\uparrow[7]$ & & Rab11 ${ }^{13}$, APC $^{14}$, LATS2 $^{15}$ & $\uparrow$ testicular germ cell tumor \\
\hline miR-129 & $\uparrow[6]$ & & LATS2 & $\downarrow$ colorectal ca. \\
\hline miR-203 & $\downarrow[5]$ & $\uparrow[$ our group] & $\begin{array}{l}\text { ABL1 }{ }^{16}, \text { DeltaNp63, TP53INP1, } \\
\text { SOCS3 }^{17}\end{array}$ & $\begin{array}{l}{ }^{\uparrow} \text { pancreatic ca., ovarian ca., bladder ca., lung ca. [5] } \\
\downarrow \text { HNSCC, CML }{ }^{18}, \text { ALL }^{19}\end{array}$ \\
\hline miR-205 & $\downarrow[5]$ & $\begin{array}{l}\uparrow[\text { our group] } \\
\downarrow[5]\end{array}$ & $\begin{array}{l}\text { ZEB1, ZEB2 }{ }^{20}, \text { E2F1, E2F5, HER3 }{ }^{21} \text {, } \\
\text { ERBB3 }^{22}, \text { PRKCE }^{23}, \text { LRP1 }^{24}\end{array}$ & $\begin{array}{l}\uparrow \text { HNSCC [16], SCC of lung [17] } \\
\downarrow \text { breast ca. [22], prostate ca. [10] }\end{array}$ \\
\hline miR-10a & & $\downarrow[$ our group $]$ & $\begin{array}{l}\text { HOXA3 }^{25}, \text { HOXB1, HOXB3 } \\
\text { HOXD } 4, \text { HOXD10 }\end{array}$ & $\begin{array}{l}\uparrow \text { HNSCC, urothelial ca. [29] } \\
\downarrow \text { colon ca. }\end{array}$ \\
\hline miR-375 & $\downarrow[1]$ & & $\mathrm{PDK}^{26}$ & $\downarrow$ HNSCC, prostate ca. $\beta$-catenin mutated HCC [30] \\
\hline
\end{tabular}

$\uparrow=$ Overexpression in ESCC compared the non-cancerous counterparts; $\downarrow=$ downregulation

${ }^{1}$ Programmed cell death $4 ;{ }^{2}$ nuclear factor I/B; ${ }^{3}$ phosphatase and tensin homolog; ${ }^{4}$ tropomyosin $1 ;{ }^{5}$ cancer; ${ }^{6}$ acute myeloid leukemia; ${ }^{7}$ chronic lymphocytic leukemia; ${ }^{8}$ hepatocellular carcinoma; ${ }^{9}$ head and neck squamous cell carcinoma; ${ }^{10}$ E2F transcription factor $1 ;{ }^{11}$ tumor protein 553 inducible nuclear protein $1 ;{ }^{12}$ adult T-cell leukemia; ${ }^{13}$ Rab pro- tein $11 ;{ }^{14}$ adenomatous polyposis coli; ${ }^{15}$ large tumor suppressor, homo$\log 2 ; 16 \mathrm{c}$-abl oncogene $1 ; 17$ suppressor of cytokine signaling 3; ${ }^{18}$ chronic myeloid leukemia; ${ }^{19}$ acute lymphocytic leukemia; ${ }^{20}$ zinc finger E-box binding homeobox; ${ }^{21}$ human epidermal growth factor receptor type $3 ;{ }^{22}$ v-erb-b2 erythroblastic leukemia viral oncogene homolog 3 ; ${ }^{23}$ protein kinase $\mathrm{C}$, epsilon; ${ }^{24}$ low-density lipoprotein receptor-related protein $1 ;{ }^{25}$ homeobox; ${ }^{26}$ phosphoinositide-dependent protein kinase-1.
They can function as oncogenes or tumor suppressors [4]. However, there has been little information on functional roles of miRNAs specific for esophageal cancer. We sought to identify miRNAs which could be specifically expressed and exert distinct biological actions in ESCC cells.

\section{miRNA Expression and ESCC in the Literature}

There have been several studies on the relationship between miRNA expression and ESCC (table 1). Feber et al. [5] reported that small RNAs isolated from 35 frozen esophageal specimens of patients with ESCC and esophageal adenocarcinoma were analyzed using the comprehensive microarray method. As a result, miR-203 and -205 were expressed as being 2- to 10 -fold lower in squamous cell carcinoma and adenocarcinomas than in normal epithelium. On the other hand, the miR-21 expression was 3- to 5 -fold higher in both tumors than in normal epithelium. In another report on a microarray basis validating 70 ESCC patients, elevated miR-21 and reduced miR-375 expression were shown in ESCC tissue compared with non-cancerous tissue [1]. Ogawa et al. [6] reported that for the association of miRNAs with prognosis, the high expression levels of 6 of the 72 miRNAs assessed by quantitative reverse transcriptase PCR (RTPCR) correlated with significantly lower survival rates. In particular, the overexpression of miR-129 was identified as a significant and independent prognostic factor in surgically treated ESCC patients. Lee et al. [7] suggested miR-373 would be a potential oncogene through suppressing large tumor suppressor homolog 2 (LATS2) expression. Hiyoshi et al. [8] reported that miR-21 was significantly elevated in ESCC tissues and cell lines. The report suggested that miR-21 could regulate cell proliferation and invasion through suppressing an apoptosisrelated molecule, programmed cell death protein 4 . In addition, elevated expression of miR-103 and -107 correlated with poor survival on multivariate analysis [9]. Table 1 summarizes the representative miRNA expression signatures in ESCC and other cancers and their corresponding predictive or confirmed targets.

\section{miR-205 and -10a Are Specific for ESCC}

We sought to determine miRNA expression signatures in ESCC cells and to assess functional roles of the specific miRNA identified. To find specific miRNAs for ESCC cells, total RNAs were extracted from OE21 and 


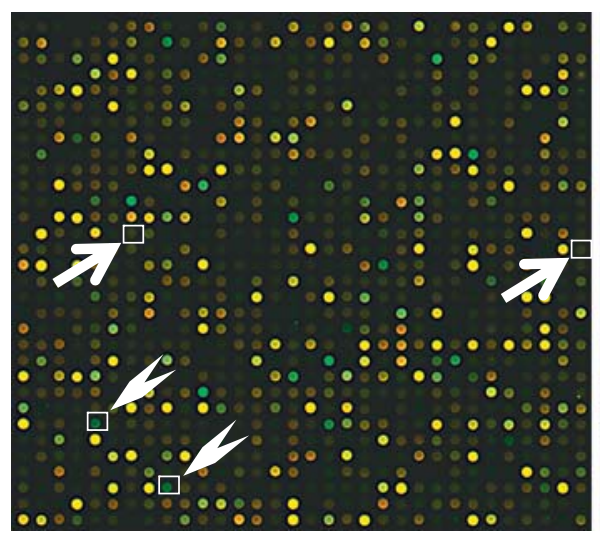

a

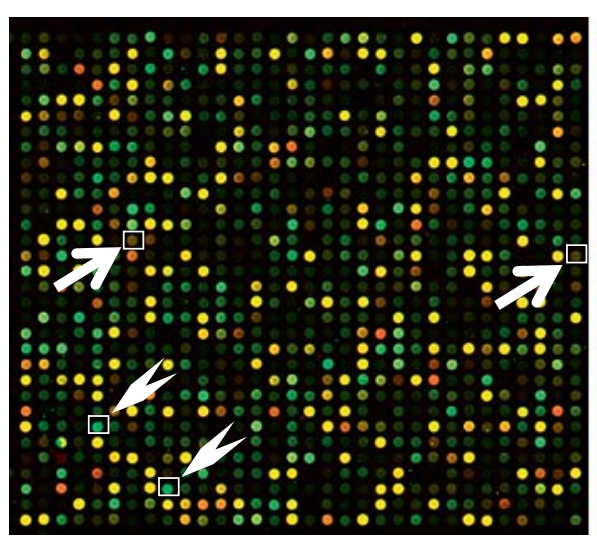

Het1A

b

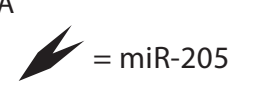

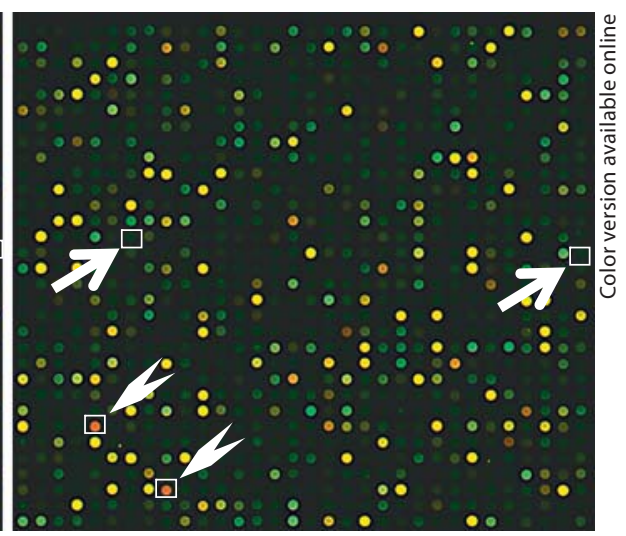

OE21

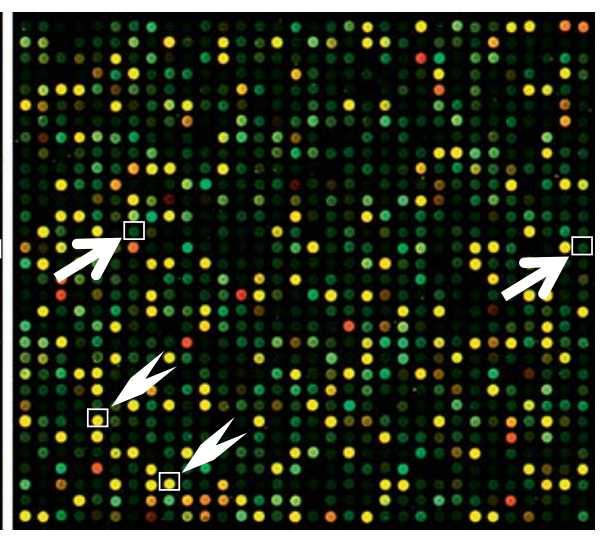

TE10 non-malignant esophageal squamous cell line, Het1A vs. ESCC cell lines, OE21 (a) or TE10 (b).

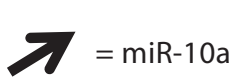

TE10 cells, representative of well- or moderately-differentiated human ESCC cell lines, respectively, and a nonmalignant human esophageal squamous cell line, Het1A. The isolated RNA samples were subjected to the comprehensive analysis of miRNA expression patterns with microarray-based technology. As a result, miR-203, -429, $-205,-200$ c and -141 were significantly (more than 2 -fold) overexpressed in both the OE21 and TE10 ESCC cells compared to Het1A cells. On the other hand, miR-153, $-100,-125 b,-10 a,-99 a,-376 a,-379,-651$ and $-146 b$ were listed in the significantly reduced miRs in expression in the two ESCC cell lines than Het1 A cells (fig. 1).

Thus, real-time RT-PCR was employed to quantify expression levels of miRNAs that showed significant differences based on the microarray results analyzed using various human malignant cell lines including ESCC and non-malignant Het1A. We used five human ESCC cell lines (TE5, TE8, TE10 and TE11 and OE21), two human Barrett adenocarcinoma cell lines (Bic-1 and Seg-1), three human gastric adenocarcinoma cell lines (AGS, AZ521 and KATOIII), two colorectal adenocarinoma cell lines (Caco-2 and DLD1), a human cervix epitheloid carcinoma cell line (HeLa), a human lung adenocarcinoma cell line (A549), and human hematological malignant cell lines (acute promyelotic leukemia, HL60; human T-cell lymphoblast-like cell line, Jurkat, and histocytic lymphoma, U937). Relative expression was calculated using U6B as the endogenous control for data normalization. Among the significantly altered miRNAs, only the miR-205 and -10 a expression levels were substantially increased and decreased, respectively, in all the ESCC cell lines compared to Het1A cells. Moreover, the miR-205 expression levels were exclusively increased in each ESCC cell line than those in the other malignant cell types examined, and hence we sought to determine the functional roles of miR-205 in ESCC. On the other hand, the miR-10a expression levels were not necessarily decreased in only the 
ESCC cells, but the reduction in expression was distinct in ESCC compared to the esophageal adenocarcinoma.

\section{miR-205 Is Associated with Cellular Migration}

but Not with Cell Proliferation, Apoptosis and

\section{Differentiation}

Transfection of anti-miR-205 inhibitor with sufficient concentrations to decrease the miR-205 expression levels had no significant impact on cellular proliferation assessed by MTS [3-(4,5-dimethylthiazol-2-yl)-5-(3carboxymethoxyphenyl)-2-(4-sulfophenyl)-2H-tetrazolium] assays. Again, there were no significant differences in degrees of apoptotic cells between the OE21 cells transfected with anti-miR-205 inhibitor or control oligonucleotides. On the other hand, knockdown of miR-205 by transfection with anti-miR-205 inhibitor significantly increased the invaded cell numbers assessed by Matrigel migration assay, while overexpression of miR-205 by miR-205 precursor transfection could inhibit the transmembrane ability significantly, indicating that miR-205 might modulate cellular migration/invasion of ESCCs. Recently it has been demonstrated that miR-205 can affect epithelial-mesenchymal transition (EMT) by targeting zinc finger E-box binding homeobox (ZEB) 1 and ZEB2. ZEB1 and ZEB2 are related homeodomain-containing transcriptional factors that repress E-cadherin transcription [10]. Knockdown of miR-205 by antimiR-205 inhibitor transfection suppressed cellular expression of ZEB2 but not ZEB1 in OE21 cells. As ZEBs are known as transcriptional repressors of E-cadherin, the downregulation of miR-205 decreased cellular E-cadherin expression, and instead, $\mathrm{N}$-cadherin appeared in the OE21 cells transfected with anti-miR-205 inhibitor, indicating acquisition of the EMT phenotype. In fact, previous studies employing reporter gene assay confirmed miR-205 binding to the ZEB2 3'-UTR [11, 12]. Using miRNA target prediction algorithms, such as ErB3, $\mathrm{E} 2 \mathrm{~F} 1$, and E2F5, ZEB1, ZEB2 and protein kinase $\mathrm{C} \varepsilon$ have been identified for putative miR-205 targets [10]. E-cadherin is a central component of the adherens junction complex responsible for cell-cell adhesion and maintenance of cytoskeleton organization. It is known that loss of E-cadherin expression is a key event of EMT, which can be recapitulated during tumor progression, constituting an early step in metastasis of tumors $[13,14]$. Gregory et al. $[11,13]$ described that the miRNA-200 family (miR-200a, -200b, -200c, -141 and -429) as well as miR205 were markedly downregulated in breast and colon cancer cells that had undergone EMT. Collectively, miR205 , along with members of the miR-200 family, can be a key regulator of EMT to enforce the indolent epitheliallike phenotype extensively and not only limited to ESCC. There is no information on the relationship between miR-205 and cancer metastasis as a phenomenon of EMT in ESCC. As referred to hereinafter, however, it has been reported that tumor metastasis signatures are associated with low expression of miR-205 in other types of cancer, such as breast cancer. In addition, lower levels of miR-205 were significantly associated with locoregional recurrence and poor survival of patients with head and neck squamous cell carcinoma [15]. Further studies are warranted to assess whether the miR-205 expression levels could be a predictive biomarker for clinical outcomes in ESCC.

To analyze the correlation between miR-205 and differentiation and ESCC in vivo, total RNAs extracted from the neoplastic and adjacent non-neoplastic samples (esophagoscopic biopsies) were subjected to real-time quantitative RT-PCR for quantification of the miR-205 expression levels. There were no significant differences in the relative miR-205 expression levels between the ESCC tumors and their adjacent non-neoplastic tissues. Next, we assessed the relationship between the miR-205 expression levels in tumor tissues and their histological subclasses of ESCC differentiation. As a result, the miR-205 expression levels did not differ among the histological ESCC differentiations. The miR-205 expression levels did not differ among the histological subclasses of ESCC differentiation.

\section{miR-205 Expression in Other Cancers}

We found that miR-205 was exclusively overexpressed in ESCC. The miR-205 expression levels were higher in ESCC cells than any of the other cell lines derived from different malignancies. It was reported that miR-205 could be a discriminator between esophageal squamous and metaplastic epithelium (Barrett's esophagus) [5]. Tran et al. [16] conducted profiling of miRNA expression in human head and neck squamous cancer cell lines, and detected 33 highly and 22 lowly expressed miRNAs. Among them, miR-205 and -212 were listed in the highest miRNAs in expression [16]. Another study identified miR-205 as one of a set of six miRNAs which were differentially expressed in pulmonary squamous cell lung carcinoma compared to adenocarcinoma [17]. These data are in agreement with previous reports that miR-205 was abundant in squamous cells in humans $[17,18]$. Approximately one-third of miRNA will exhibit substantial tissue specificity. miR-205 exhibits high endogenous expression in a broad range of tissues containing squamous epithe- 
Fig. 2. A schematic figure of the regulatory networks as for miR-205 and miR-10a. $\mathrm{RA}=$ Retinoic acid; $\mathrm{RARinh}=$ retinoic acid receptor inhibitor; HOX = homeobox; $\mathrm{ZEB}=$ zinc finger E-box binding homeobox; EMT = epithelial-mesenchymal transition; E2F1 = E2F transcription factor 1; E2F5 = E2F transcription factor 5; HER3 = human epidermal growth factor receptor type 3; ERBB3 = v-erb-b2 erythroblastic leukemia viral oncogene homolog 3; $\mathrm{PRKCE}=$ protein kinase $\mathrm{C} \varepsilon$; LRP1 = lowdensity lipoprotein receptor-related protein 1; MUC1 = mucin 1; cell surface associated; JUP = junction plakoglobin.

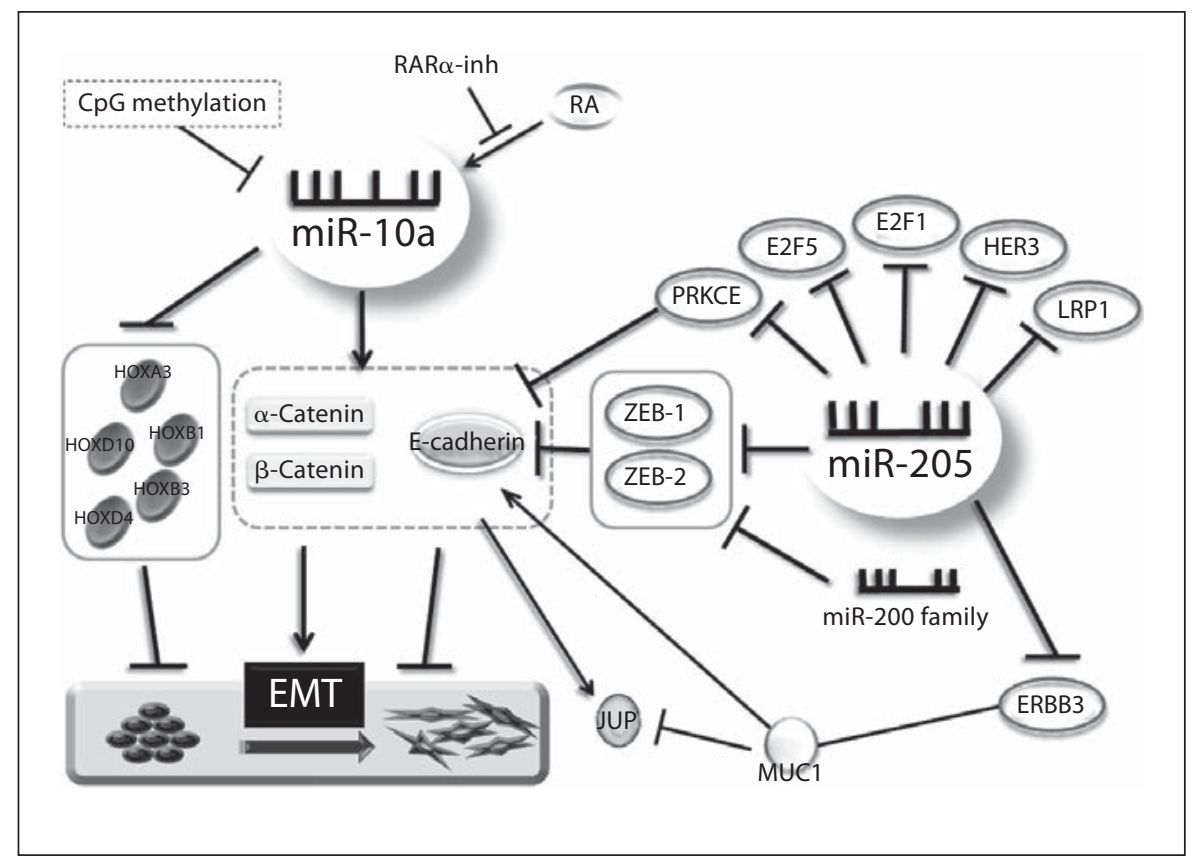

lia, especially in the skin, esophagus and trachea [19]. miR-205 is a highly conserved miRNA with homologs in diverse species [17, 20, 21]. In zebrafish, miR-205 is predominantly expressed in the epidermis, while in the mouse it was detected in the footpad, tongue, epidermis and corneal epithelium, but not the small intestine, brain, heart, liver, kidney and spleen $[20,21]$. These observations suggest that miR-205 might represent a stratified squamous epithelium miRNA.

On the other hand, miRNA profiling revealed that miR-205 expression was downregulated in some other types of malignancies such as breast and prostate cancer $[10,22,23]$. Iorio et al. [22] reported that miR-205 was significantly underexpressed in breast tumors compared with matched normal mammary tissue. Furthermore, breast cancer cell lines expressed lower levels of miR-205 than the non-malignant mammary cells examined in their study. Of note, ectopic expression of miR-205 significantly inhibited cell proliferation and anchorage-independent growth in breast cancer cells possibly via targeting HER (human epidermal growth factor receptor) [22]. In this context, miR-205 could interfere with the phosphatidylinositol-3-kinase/Akt survival pathway mediated by HER [22]. Similar to our observations, miR-205 was found to function as a tumor suppressor in diverse cell types $[10,13,22]$. Enforced expression of miR-205 was shown to inhibit cell invasion and suppress lung metastasis of breast cancer cells in nude mice possibly through targeting ErbB3 [23]. MiR-205 also exerts inhibitory effects on cellular invasiveness and migration in prostate cancer and glioblastoma cells, through downregulation of the protein kinase $\mathrm{C} \varepsilon$ and low-density lipoprotein receptor-related protein 1 , respectively $[10,24]$. In the present study, knockdown of miR-205 expression substantially reduced cellular expression of ZEB2 in ESCC cells.

\section{miR-10a Expression in Cancers}

miR-10a was recently reported as being a suppressor of homeobox (HOX) genes in several cancers, including SCCs. Although HOX genes are generally known to determine morphology on the anteroposterior axis of bilaterian animals, HOX genes that play a role in tumorgenesis or metastasis are beginning to be understood. Shen et al. [25] reported miRNA profiles of malignantly transformed human bronchial epithelial cells, 16HBE-T, induced by anti-benzo[a]pyrene-trans-7,8-diol-9,10-epoxide (anti-BPDE). MiR-10a was differentially expressed in the malignant $16 \mathrm{HBE}-\mathrm{T}$, suggesting a potential role of miR-10a in cell transformation induced by anti-BPDE. HOXA1 was similarly upregulated in the malignant $16 \mathrm{HBE}-\mathrm{T}$, suggesting that miR-10a is associated with the process of HOXA1-mediated transformation. Weiss et al. [26] reported that miR-10a was a retinoic acid-responsive mediator of invasiveness and metastatic behavior in pancreatic cancer through suppression of HOXB1 and HOXB3. 
Epigenetic silencing via $\mathrm{CpG}$ island hypermethylation of the promoter region of certain tumor suppressor genes is related in cancer pathogenesis. Tan et al. [27] described that miR-10a-induced inhibition of transcription is associated with DNA methylation of Hoxd4 promoter. In a colon cancer cell line HCT116, miR-10a expression was significantly reduced and it was significantly increased in the DNA methyltransferase- 1 and -3 b double knockout HCT116 cells. In line with this, CpG islands were found to be located within 3-kb upstream of the miR-10a gene [28]. Similarly, miR-10a expression was restored in OE21 cells treated with 5-aza-2'-deoxycytidine, a demethylating agent, suggesting epigenetic regulation of this miRNA in many cancer cell types including colorectal cancer and ESCC. In addition, miR-10a may regulate EMT through targeting and hence translationally inhibiting HOXs (fig. 2), suggesting that miR-10a may function as a tumor suppressor.

\section{Conclusion}

In conclusion, miR-205 and miR-10a expression was significantly altered in the ESCC cells examined. MiR205 is likely to control cell invasion and migration in ESCC cells through its repression of ZEB2, a repressor of E-cadherin. Similar suppressive mechanisms of metastatic behavior of the cancer cells may fit the possible role of miR-10a, as it targets HOX genes. MiR-205 and -10a may be involved in the ESCC pathogenesis.

\section{Disclosure Statement}

The authors declare that no functional or conflict of interests exists in relation to the content of the article.

\section{References}

1 Mathe EA, Nguyen GH, Bowman ED, Zhao Y, Budhu A, Schetter AJ, Braun R, Reimers M, Kumamoto K, Hughes D, Altorki NK, Casson AG, Liu CG, Wang XW, Yanaihara N, Hagiwara N, Dannenberg AJ, Miyashita M, Croce CM, Harris CC: MicroRNA expression in squamous cell carcinoma and adenocarcinoma of the esophagus: associations with survival. Clin Cancer Res 2009; 15: 6192-6200.

$\checkmark 2$ Fareed KR, Kaye P, Soomro IN, Ilyas M, Martin S, Parsons SL, Madhusudan S: Biomarkers of response to therapy in oesophago-gastric cancer. Gut 2009;58:127-143.

- 3 Rosenfeld N, Aharonov R, Meiri E, Rosenwald S, Spector Y, Zepeniuk M, Benjamin H, Shabes N, Tabak S, Levy A, Lebanony D, Goren Y, Silberschein E, Targan N, Ben-Ari A, Gilad S, Sion-Vardy N, Tobar A, Feinmesser M, Kharenko O, Nativ O, Nass D, Perelman M, Yosepovich A, Shalmon B, Polak-Charcon S, Fridman E, Avniel A, Bentwich I, Bentwich Z, Cohen D, Chajut A, Barshack I: MicroRNAs accurately identify cancer tissue origin. Nat Biotechnol 2008;26:462-469.

$\checkmark 4$ Croce CM: Causes and consequences of microRNA dysregulation in cancer. Nat Rev Genet 2009;10:704-714.

5 Feber A, Xi L, Luketich JD, Pennathur A, Landreneau RJ, Wu M, Swanson SJ, Godfrey TE, Litle VR: MicroRNA expression profiles of esophageal cancer. J Thorac Cardiovasc Surg 2008;135:255-260.
-6 Ogawa R, Ishiguro H, Kuwabara Y, Kimura M, Mitsui A, Katada T, Harata K, Tanaka T, Fujii Y: Expression profiling of micro-RNAs in human esophageal squamous cell carcinoma using RT-PCR. Med Mol Morphol 2009;42:102-109.

7 Lee KH, Goan YG, Hsiao M, Lee CH, Jian SH, Lin JT, Chen YL, Lu PJ: MicroRNA-373 (miR373) post-transcriptionally regulates large tumor suppressor, homolog 2 (LATS2), and stimulates proliferation in human esophageal cancer. Exp Cell Res 2009;315:2529-2538.

-8 Hiyoshi Y, Kamohara H, Karashima R, Sato N, Imamura Y, Nagai Y, Yoshida N, Toyama E, Hayashi N, Watanabe M, Baba H: MicroRNA-21 regulates the proliferation and invasion in esophageal squamous cell carcinoma. Clin Cancer Res 2009;15:1915-1922.

$\checkmark 9$ Guo Y, Chen Z, Zhang L, Zhou F, Shi S, Feng X, Li B, Meng X, Ma X, Luo M, Shao K, Li N, Qiu B, Mitchelson K, Cheng J, He J: Distinctive microRNA profiles relating to patient survival in esophageal squamous cell carcinoma. Cancer Res 2008;68:26-33.

10 Gandellini P, Folini M, Longoni N, Pennati M, Binda M, Colecchia M, Salvioni R, Supino R, Moretti R, Limonta P, Valdagni R, Daidone MG, Zaffaroni N: miR-205 exerts tumor-suppressive functions in human prostate through down-regulation of protein kinase C $\varepsilon$. Cancer Res 2009;69:2287-2295.

-11 Gregory PA, Bert AG, Paterson EL, Barry SC, Tsykin A, Farshid G, Vadas MA, KhewGoodall Y, Goodall GJ: The miR-200 family and miR-205 regulate epithelial to mesenchymal transition by targeting ZEB1 and SIP1. Nat Cell Biol 2008;10:593-601.
12 Korpal M, Lee ES, Hu G, Kang Y: The miR200 family inhibits epithelial-mesenchymal transition and cancer cell migration by direct targeting of E-cadherin transcriptional repressors ZEB1 and ZEB2. J Biol Chem 2008;283:14910-14914

13 Gregory PA, Bracken CP, Bert AG, Goodall GJ: MicroRNAs as regulators of epithelialmesenchymal transition. Cell Cycle 2008; 7 : 3112-3118.

14 Thiery JP, Sleeman JP: Complex networks orchestrate epithelial-mesenchymal transitions. Nat Rev Mol Cell Biol 2006;7:131-142.

15 Childs G, Fazzari M, Kung G, Kawachi N, Brandwein-Gensler M, McLemore M, Chen Q, Burk RD, Smith RV, Prystowsky MB, Belbin TJ, Schlecht NF: Low-level expression of microRNAs let-7d and miR-205 are prognostic markers of head and neck squamous cell carcinoma. Am J Pathol 2009;174:736-745.

16 Tran N, McLean T, Zhang X, Zhao CJ, Thomson JM, O'Brien C, Rose B: MicroRNA expression profiles in head and neck cancer cell lines. Biochem Biophys Res Commun 2007; 358:12-17.

$17 \mathrm{Wu}$ H, Mo YY: Targeting miR-205 in breast cancer. Expert Opin Ther Targets 2009;13: 1439-1448.

18 Ryan DG, Oliveira-Fernandes M, Lavker RM: MicroRNAs of the mammalian eye display distinct and overlapping tissue specificity. Mol Vis 2006;12:1175-1184

19 Fletcher AM, Heaford AC, Trask DK: Detection of metastatic head and neck squamous cell carcinoma using the relative expression of tissue-specific miR-205. Transl Oncol 2008;1:202-208. 
-20 Ason B, Darnell DK, Wittbrodt B, Berezikov E, Kloosterman WP, Wittbrodt J, Antin PB, Plasterk RH: Differences in vertebrate microRNA expression. Proc Natl Acad Sci USA 2006;103:14385-14389.

-21 Shingara J, Keiger K, Shelton J, LaosinchaiWolf W, Powers P, Conrad R, Brown D, Labourier E: An optimized isolation and labeling platform for accurate microRNA expression profiling. RNA 2005;11:1461-1470.

-22 Iorio MV, Casalini P, Piovan C, Di Leva G, Merlo A, Triulzi T, Menard S, Croce CM, Tagliabue E: MicroRNA-205 regulates HER3 in human breast cancer. Cancer Res 2009;69: 2195-2200.

-23 Wu H, Zhu S, Mo YY: Suppression of cell growth and invasion by miR-205 in breast cancer. Cell Res 2009;19:439-448.
24 Song H, Bu G: MicroRNA-205 inhibits tumor cell migration through down-regulating the expression of the LDL receptor-related protein 1. Biochem Biophys Res Commun 2009;388:400-405.

25 Shen YL, Jiang YG, Greenlee AR, Zhou LL, Liu LH: MicroRNA expression profiles and miR-10a target in anti-benzo[a]pyrene-7,8-diol-9,10-epoxide-transformed human $16 \mathrm{HBE}$ cells. Biomed Environ Sci 2009;22:14-21.

26 Weiss FU, Marques IJ, Woltering JM, Vlecken DH, Aghdassi A, Partecke LI, Heidecke CD, Lerch MM, Bagowski CP: Retinoic acid receptor antagonists inhibit miR-10a expression and block metastatic behavior of pancreatic cancer. Gastroenterology 2009;137 2136-2145.
27 Tan Y, Zhang B, Wu T, Skogerbo G, Zhu X, Guo X, He S, Chen R: Transcriptional inhibition of Hoxd4 expression by miRNA-10a in human breast cancer cells. BMC Mol Biol 2009;10:12.

28 Han L, Witmer PD, Casey E, Valle D, Sukumar S: DNA methylation regulates microRNA expression. Cancer Biol Ther 2007;6: 1284-1288.

29 Wong TS, Liu XB, Chung-Wai Ho A, PoWing Yuen A, Wai-Man Ng R, Ignace Wei W: Identification of pyruvate kinase type M2 as potential oncoprotein in squamous cell carcinoma of tongue through microRNA profiling. Int J Cancer 2008;123:251-257.

30 Osaki M, Takeshita F, Ochiya T: MicroRNAs as biomarkers and therapeutic drugs in human cancer. Biomarkers 2008;13:658-670. 